\title{
Women of reproductive age in a tertiary intensive care unit: indications, outcome and the impact of pregnancy-a retrospective cohort study
}

\author{
Karishma P. Ramlakhan ${ }^{1 *}$, Diederik Gommers ${ }^{2}$, Carmen E. R. M. Jacobs ${ }^{3}$, Khaoula Makouri ${ }^{3}$, \\ Johannes J. Duvekot ${ }^{3}$, Irwin K. M. Reiss ${ }^{4}$, Arie Franx ${ }^{3}$, Jolien W. Roos-Hesselink and Jérôme M. J. Cornette ${ }^{3}$
}

\begin{abstract}
Background: To evaluate the indications for admission and mortality rates of women of reproductive age admitted to a tertiary Intensive Care Unit (ICU) and to compare the outcomes of obstetric and non-obstetric admissions.

Methods: A retrospective cohort study was performed, including all women aged 17-41 years admitted to a level 3 ICU in the Netherlands, between January 1, 2000 and January 1, 2016. Primary outcome was indication for admission and mortality. Mortality, length of stay (LOS), need for mechanical ventilation and APACHE II score were compared between obstetric and non-obstetric admissions. The obstetric group was further analyzed for maternal and perinatal outcomes.

Results: 3461 women (median age 32 years) were included, with an overall mortality rate of $13.3 \%$. The obstetric group consisted of 265 women $(7.7 \%)$. The non-obstetric group $(n=3196)$ was admitted most often for cardiovascular disease (19.6\%), followed by oncologic disease (15\%). Mortality was the highest in women with oncologic disease (23.9\%). The obstetric group had lower mortality compared to the non-obstetric group ( $4.9 \%$ vs. $14 \%, p<0.001)$, despite higher APACHE II score (14 vs. $11, p<0.001)$ and a higher ventilation rate $(47.9 \%$ vs. $39 \%, p=0.004)$. Major surgical or endovascular interventions, besides caesarean section, were performed in $46 \%$ of the obstetric group. Perinatal death occurred in $17.2 \%$ and of the surviving infants, $63.2 \%$ were born preterm and $45.1 \%$ required Neonatal Intensive Care Unit admission.

Conclusions: Cardiovascular disease is the most important indication for admission and oncologic disease is associated with highest mortality in women of reproductive age. Obstetric patients constitute a small percentage of all ICU admissions in a tertiary ICU center. They have lower mortality rates than non-obstetric young female patients, despite a more severe initial presentation. Nevertheless lasting maternal morbidity and perinatal mortality and morbidity is frequent.
\end{abstract}

Keywords: Critical care, Pregnancy, Mortality, Women's health

*Correspondence: k.ramlakhan@erasmusmc.nl

1 Department of Cardiology, Erasmus MC, University Medical Center

Rotterdam, PO Box 2040, 3000 CA Rotterdam, The Netherlands

Full list of author information is available at the end of the article

\section{Background}

The average Intensive Care Unit (ICU) patient is male, $>60$ years of age and has significant comorbidity and multi-organ involvement $[1,2]$. Most young women of reproductive age are considered in the prime of their life. 
They are far less likely to require admission in an ICU unit and are therefore less studied. Very little is known on indications for ICU admission and outcome in young women of reproductive age. Obstetric patients form a particular subgroup of this population. A recent survey in Australia and new Zealand showed that obstetric patients formed $1.3 \%$ of the total ICU population and $11 \%$ of the young women of reproductive age in the ICU [3]. Pregnancy specific problems like pre-eclampsia, postpartum hemorrhage or deterioration of pre-existent conditions can be sudden and life threatening, requiring immediate intensive management and monitoring [4, 5]. Most obstetricians are less familiar or lack facilities for this critical management, requiring ICU admission for these patients. Prompt and skilled interventions can rapidly counter most of these life-threatening complications. Two large nationwide studies showed that mortality for obstetric women admitted to ICU is low (0.7-1.7\%) [3, 6]. Tertiary referral centers with high case load therefore invest in Obstetric High Care Units, permitting the management of most of these life threatening problems in an obstetric setting. ICU admission is then reserved for more complicated cases requiring specific critical care expertise.

Our aim was to analyze indications for admissions and respective mortality in young women of reproductive age in a tertiary care referral ICU. We compared outcomes between obstetric and non-obstetric admissions and described maternal and perinatal outcome in this selected population.

\section{Methods}

We performed a retrospective cohort study in the Erasmus MC, University Medical Center Rotterdam in the Netherlands, which is the regional tertiary referral center for both obstetric and intensive care medicine. The study period ranged from January 1, 2000 until January 1, 2016. The study was approved by the Erasmus MC Institutional Review Board and the need for informed consent was waived. The handling of personal data complied with the Dutch Personal Data Protection Act; data were de-identified and handled confidentially.

In addition to the regular ward for level 1 obstetric medical care, the hospital is equipped with a level 2 Obstetric High Care Unit [7]. This dedicated unit is managed round the clock on-site by a specialized obstetrical staff, including a maternal-fetal medicine specialist, neonatologist and obstetric anesthesiologist, in close collaboration with other obstetric medicine specialists and consultants. These facilities permit continuous hemodynamic monitoring and care by trained obstetric nurses. ICU are separate wards, providing level 3 care in a closed system. Most high-risk obstetric complications like severe preeclampsia, postpartum hemorrhage (PPH) or sepsis are managed in the level 2 Obstetric High Care Unit. Admission or transfer from the level 1 or 2 wards to a level 3 ICU unit is decided for clinical reasons in communication between the obstetric caregiver and the ICU physician. Criteria for ICU admission are severe multiorgan involvement, the necessity of invasive ventilation, renal dialysis or inotropic support $[7,8]$.

The ICU data warehouse was screened for admissions and to collect data of young women between 17 and 41 years requiring level 3 intensive care in the aforementioned time period. The obstetric group was crosschecked with datasets from the Obstetrics department, and medical records were individually reviewed to collect data. Inclusion criteria for the obstetric group were all women requiring level 3 ICU care during pregnancy or $\leq 6$ weeks postpartum.

ICD-10 codes were used to determine the indications for ICU admission, which were divided in 10 subgroups: obstetric, cardiovascular, pulmonary, gastrointestinal, neurological or neurovascular, internal medicine, surgical, oncologic, congenital (all types) or other disease. Outcomes included the APACHE II score, ICU and hospital LOS, mortality within one year and need for mechanical ventilation. The APACHE II score is a prognostic model for ICU mortality and serves as a classification for the severity of disease on admission [9].

Descriptive parameters for the obstetric admissions were parity, initial level of obstetric care, mode of delivery and moment of admission in the ICU. Indications for ICU admission were defined as direct obstetric (e.g. $\mathrm{PPH}$ ), indirect obstetric (decompensation of underlying disease due to pregnancy) and non-obstetric pathology (unrelated to pregnancy). Data on the need for mechanical ventilation and need for intervention were collected. Interventions were defined as surgical (excluding caesarean section), endovascular or invasive medical interventions such as extracorporeal membrane oxygenation (ECMO).

Perinatal outcomes included gestational age, birthweight, 5 min Apgar score, umbilical cord $\mathrm{pH}$, Neonatal Intensive Care Unit (NICU) admission, respiratory support, mortality (intrauterine, intrapartum or postpartum) and composite neonatal morbidity. Composite neonatal morbidity was defined as respiratory distress syndrome, necrotizing enterocolitis, intraventricular hemorrhage, periventricular leukomalacia and/or sepsis confirmed by blood culture in neonates born at $\geq 24$ weeks of gestation, which is considered the limit of viability in the Netherlands [4].

One-year mortality rate was calculated for the entire cohort and per subgroup, after which the mortality rate of the obstetric admissions was compared to the 
pooled mortality of the non-obstetric admissions. LOS, APACHE II score and ventilator support rate were also compared between the obstetric and non-obstetric admissions. The detailed maternal and fetal characteristics and outcomes in the obstetric group were compared between antepartum and postpartum maternal ICU admissions. Continuous data are presented as mean with standard deviation (SD), or as median (Q1-Q3) when skewed. Differences between the groups were calculated using Student's t-tests or Mann-Whitney tests as appropriate. Categorical data are presented as percentages and compared using $\chi^{2}$ tests. Significance was set at $p<0.05$. Statistical analysis was carried out using IBM SPSS Statistics 21.0 (IBM New York, USA).

\section{Results}

During the 15-year study period, 3461 female ICU admissions in 3039 women between 17 and 41 years of age were recorded. Indications for ICU admission and associated mortality rates are presented in Fig. 1. The group of admissions due to cardiovascular disease was the largest (19.6\%), followed by oncologic disease (15\%) and neurologic disease (14.2\%). The group of obstetric admissions consisted of 265 admissions in 265 women (7.7\%). The overall mortality rate of all 3461 female admissions was $13.3 \%$. The subgroup with oncologic disease had the highest mortality rate of $23.9 \%$, whereas the mortality rate in the obstetric group was the second lowest at $4.9 \%$.

Table 1 presents the comparison between obstetric $(\mathrm{n}=265)$ and non-obstetric admissions $(\mathrm{n}=3196)$.
Table 1 Outcomes of the obstetric group and the non-obstetric group

\begin{tabular}{lccr}
\hline & $\begin{array}{l}\text { Obstetric group } \\
\mathbf{n = 2 6 5}\end{array}$ & $\begin{array}{l}\text { Non-obstetric } \\
\text { group } \\
\mathbf{n = 3 1 9 6}\end{array}$ & $\boldsymbol{p}$ value \\
\hline Age (years) & $31(28-35)$ & $32(25-37)$ & 0.195 \\
Mortality & $13(4.9 \%)$ & $446(14.0 \%)$ & $<0.001$ \\
APACHE II score & $14(9-19)$ & $11(6-17)$ & $<0.001$ \\
Mechanical ventila- & $127(47.9 \%)$ & $1247(39 \%)$ & 0.004 \\
$\quad$ tion & & $10(5-20)$ & 0.060 \\
Hospital LOS (days) & $11(6.5-19)$ & $2(2-4)$ & 0.343 \\
ICU LOS (days) & $2(2-4)$ & &
\end{tabular}

Data in $\mathrm{n}(\%)$ and median (Q1-Q3)

LOSlength of stay

While APACHE II score (14 vs. $11, p<0.001)$ and use of invasive ventilation $(47.9 \%$ vs. $39 \%, p=0.004)$ were significantly higher, one-year mortality was lower $(4.9 \%$ vs. $11 \%, p<0.001)$ in the obstetric group. ICU LOS and hospital LOS were not different between obstetric and nonobstetric admissions.

The obstetric baseline characteristics and admission diagnosis are further described in Table 2. Most obstetric ICU admissions (median age 31, range 17-41) took place in the immediate postpartum period $(\mathrm{n}=198,74.7 \%)$. Antepartum admissions $(\mathrm{n}=67,25.3 \%)$ occurred at a median of 28 weeks of gestation (Q1-Q3 $24^{1 / 7}-33^{1 / 7}$ ). Direct obstetric complications were the most common cause for ICU admission (72.5\%), followed by indirect

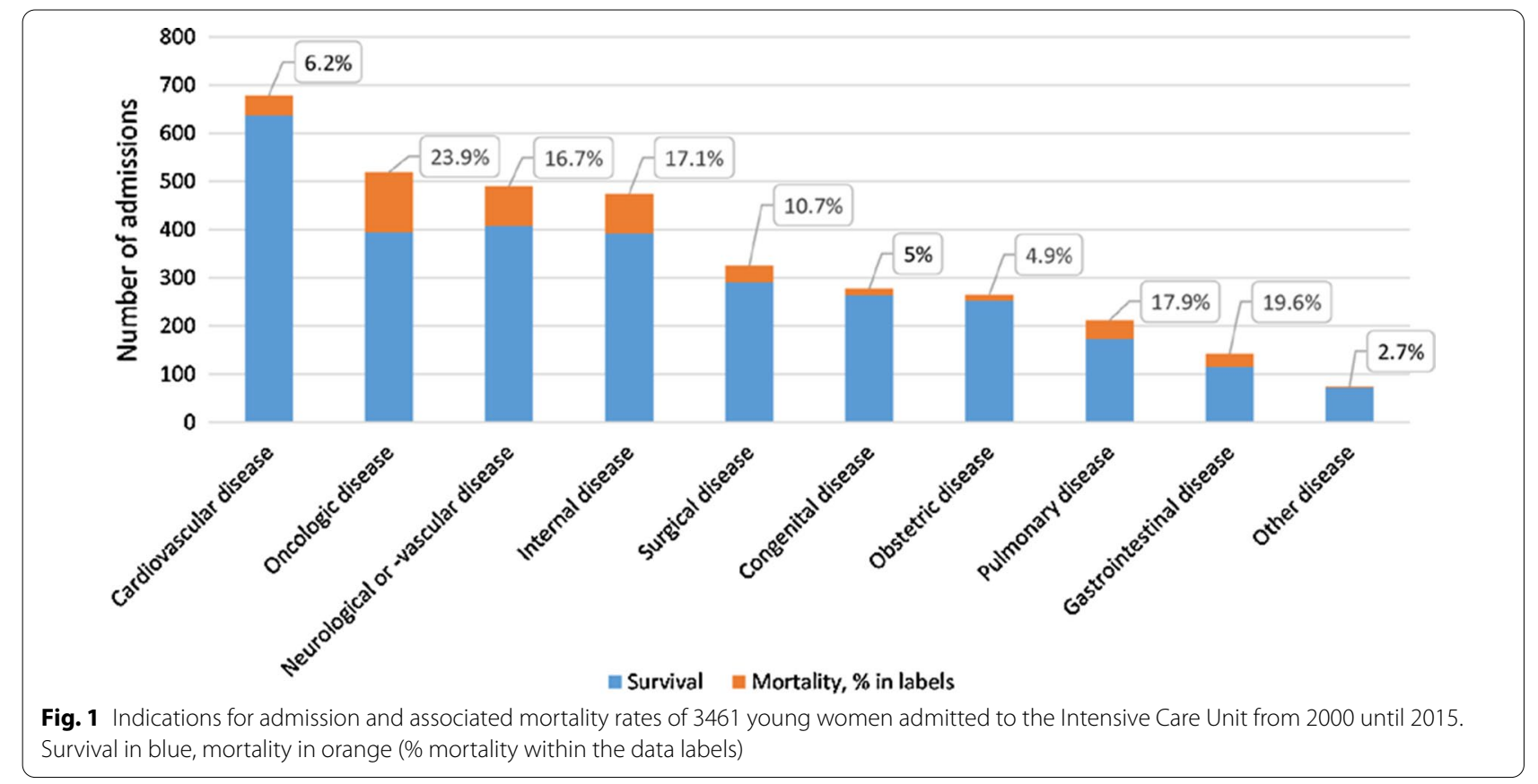


Table 2 Baseline characteristics in the obstetric group at admission to ICU

\begin{tabular}{|c|c|c|c|c|}
\hline Baseline characteristics & $\begin{array}{l}\text { All obstetric } \\
\text { admissions } \\
n=265\end{array}$ & $\begin{array}{l}\text { Antepartum admissions } \\
\mathrm{n}=67\end{array}$ & $\begin{array}{l}\text { Postpartum } \\
\text { admissions } \\
n=198\end{array}$ & $p$ value \\
\hline Age, years & $31(28-35)$ & $30(24-33)$ & $32(29-35)$ & 0.006 \\
\hline Nulliparity & $123(47.1 \%)$ & $36(53.7 \%)$ & $87(44.8 \%)$ & 0.209 \\
\hline \multicolumn{5}{|l|}{ Referred by } \\
\hline Primary care center & $42(16 \%)$ & $11(16.7 \%)$ & $31(15.8 \%)$ & 0.883 \\
\hline Secondary care center & $150(57.3 \%)$ & $40(60.6 \%)$ & $110(56.1 \%)$ & 0.554 \\
\hline Tertiary care center & $70(26.7 \%)$ & $15(22.7 \%)$ & $55(28.1 \%)$ & 0.387 \\
\hline Antepartum admissions & $67(25.3 \%)$ & $67(100 \%)$ & - & - \\
\hline Gestational age at admission, weeks & - & $28\left(24^{1 / 7}-33^{1 / 7}\right)$ & - & - \\
\hline$<24$ weeks & - & $11(17.5 \%)$ & - & - \\
\hline $24^{0 / 7}-31^{6 / 7}$ & - & $35(55.6 \%)$ & - & - \\
\hline $32^{0 / 7}-36^{6 / 7}$ & - & $11(17.5 \%)$ & - & - \\
\hline $37^{0 / 7}-41^{6 / 7}$ & - & $6(9.5 \%)$ & - & - \\
\hline$>42$ weeks & - & 0 & - & - \\
\hline Postpartum admissions & $198(74.7 \%)$ & - & $198(100 \%)$ & - \\
\hline Time between delivery and admission, days & - & - & $0(0-0)$ & - \\
\hline \multicolumn{5}{|l|}{ Mode of delivery } \\
\hline No delivery $(<16 \text { weeks })^{a}$ & $18(6.8 \%)$ & $12(17.9 \%)$ & $6(3 \%)$ & $<0.001$ \\
\hline Vaginal delivery & $87(32.8 \%)$ & $29(43.4 \%)$ & $58(29.3 \%)$ & 0.035 \\
\hline Caesarean section & $160(60.4 \%)$ & $26(38.8 \%)$ & $134(67.7 \%)$ & $<0.001$ \\
\hline \multicolumn{5}{|l|}{ Admission diagnosis } \\
\hline Direct obstetric & $192(72.5 \%)$ & $39(58.2 \%)$ & $153(77.3 \%)$ & 0.003 \\
\hline Haemorrhage & $93(35.1 \%)$ & $7(10.4 \%)$ & $86(43.4 \%)$ & $<0.001$ \\
\hline Hypertensive disorders & $46(17.4 \%)$ & $15(22.4 \%)$ & $31(15.7 \%)$ & 0.209 \\
\hline Sepsis & $26(9.8 \%)$ & $9(13.4 \%)$ & $17(8.6 \%)$ & 0.249 \\
\hline Other direct obstetric & $27(10.2 \%)$ & $8(11.9 \%)$ & $19(9.6 \%)$ & 0.583 \\
\hline Indirect obstetric & $56(21.1 \%)$ & $21(31.3 \%)$ & $35(17.7 \%)$ & 0.018 \\
\hline Cardiovascular & 24 (9.1\%) & $7(10.4 \%)$ & $17(8.6 \%)$ & 0.646 \\
\hline Respiratory & $11(4.2 \%)$ & $6(9 \%)$ & $5(2.5 \%)$ & 0.023 \\
\hline Cerebrovascular & $5(1.9 \%)$ & $2(3 \%)$ & $3(1.5 \%)$ & 0.445 \\
\hline Metabolic & $5(1.9 \%)$ & $2(3 \%)$ & $3(1.5 \%)$ & 0.445 \\
\hline Gastrointestinal & $5(1.9 \%)$ & $2(3 \%)$ & $3(1.5 \%)$ & 0.445 \\
\hline Auto-immune & $2(0.8 \%)$ & $0(0 \%)$ & $2(1.0 \%)$ & 0.409 \\
\hline Other indirect obstetric & $4(1.5 \%)$ & $2(3 \%)$ & $2(1 \%)$ & 0.252 \\
\hline Non-obstetric & $17(6.4 \%)$ & $7(10.4 \%)$ & $10(5.1 \%)$ & 0.119 \\
\hline Malignancy & $4(1.5 \%)$ & $1(1.5 \%)$ & $3(1.5 \%)$ & 0.990 \\
\hline Trauma & $2(0.8 \%)$ & $2(3 \%)$ & $0(0 \%)$ & 0.015 \\
\hline Other non-obstetric & $11(4.1 \%)$ & $4(6 \%)$ & $7(3.5 \%)$ & 0.388 \\
\hline
\end{tabular}

Data in $\mathrm{n}(\%)$ and median (Q1-Q3). $p$ values were calculated between antepartum admissions versus postpartum admissions

${ }^{a}$ No delivery was defined as miscarriage, elective or medically indicated termination of pregnancy or ectopic pregnancy

obstetric complications (21.1\%), among which maternal cardiovascular disease was most frequent (9.1\%). Comparing antepartum and postpartum admissions, direct obstetric causes (mainly driven by PPH) were more frequently seen in the postpartum group (58.2\% vs. $77.3 \%$, $p=0.003)$, whereas indirect obstetric causes were more frequent in the antepartum group $(31.3 \%$ vs. $17.7 \%$, $p=0.018)$. Major endovascular or surgical interventions or advanced organ support other than mechanical ventilation were performed in $46 \%$ of the obstetric group (Table 3). Uterine artery embolization was the most frequent intervention (15.8\%), followed by hysterectomy (15.5\%). 
Table 3 Maternal morbidity

\begin{tabular}{lc}
\hline Interventions & $\begin{array}{l}\text { Obstetric ICU } \\
\text { admissions } \\
\mathbf{n}=\mathbf{2 6 5}\end{array}$ \\
\hline Medical intervention only & $143(54 \%)$ \\
Uterine artery embolization (UAE) & $48(15.8 \%)$ \\
Hysterectomy & $41(15.5 \%)$ \\
Laparotomy & $27(10.2 \%)$ \\
Neurosurgery (including endovascular coiling) & $9(3.4 \%)$ \\
Gastrointestinal surgery (e.g. colostomy creation) & $9(3.4 \%)$ \\
Cardiothoracic surgery & $5(1.9 \%)$ \\
Urological surgery & $2(0.8 \%)$ \\
Limb amputation & $2(0.8 \%)$ \\
Liver transplant & $2(0.8 \%)$ \\
ECMO & $5(1.9 \%)$ \\
Haemodialysis & $14(5.3 \%)$ \\
Use of vasopressors or inotropes & $46(17.1 \%)$ \\
Mechanical ventilation & $127(47.9 \%)$ \\
\hline
\end{tabular}

Data in $\mathrm{n}(\%)$

ECMO extracorporeal membrane oxygenation

${ }^{\text {a }}$ Medical intervention only was defined as no surgical or endovascular intervention or advanced organ support, excluding mechanical ventilation
Perinatal data was unavailable for 26 pregnancies, leading to exclusion in the analysis. There were 18 twin pregnancies in remaining 239 pregnancies, and therefore outcome is described for 257 infants in Table 4. Before the limit of viability (24 weeks of gestation), there were 6 (2.3\%) spontaneous intrauterine deaths and $4(1.5 \%)$ terminations of pregnancy for severity of maternal disease. After the limit of viability, there were 26 (9.9\%) spontaneous intrauterine deaths and one $(0.4 \%)$ termination of pregnancy. There were $8(3.1 \%)$ neonatal deaths during or after delivery, making the total mortality rate $17.2 \%$. Median gestational age at delivery and birth weight were 35 weeks and $2215 \mathrm{~g}$. Preterm delivery occurred in 157 (63.2\%) pregnancies, 112 (40.7\%) of which were medically indicated preterm deliveries (iatrogenic). NICU admission was required in $45.1 \%$ of all surviving neonates, for a median duration of 8 days (range $0-177$ ). Mechanical ventilation was needed in $34.1 \%$, cardiopulmonary resuscitation was performed in $5.1 \%$ and composite neonatal morbidity was reported in $29.1 \%$ of all neonates. Mortality was higher and gestational age at birth and birth weight were lower among the infants of women admitted during pregnancy, compared to women admitted postpartum $(43.1 \%$ vs. $18 \%, p<0.001)$.

Table 4 Neonatal outcome

\begin{tabular}{|c|c|c|c|c|}
\hline & $\begin{array}{l}\text { All admissions } \\
n=257\end{array}$ & $\begin{array}{l}\text { Antepartum admissions } \\
\mathrm{n}=59\end{array}$ & $\begin{array}{l}\text { Postpartum admissions } \\
n=198\end{array}$ & $p$ value \\
\hline Male & $48.2 \%$ & $52.8 \%$ & $47.9 \%$ & 0.524 \\
\hline Female & $51.8 \%$ & $47.2 \%$ & $52.1 \%$ & \\
\hline Gestational age at birth, weeks & $35\left(30^{2 / 7}-38^{1 / 7}\right)$ & $31^{4 / 7}\left(27^{4 / 7}-36^{6 / 7}\right)$ & $36\left(31^{4 / 7}-38^{4 / 7}\right)$ & $<0.001$ \\
\hline$<24$ weeks & $5.6 \%$ & $12.5 \%$ & $2.8 \%$ & \\
\hline $24^{0 / 7}-31^{6 / 7}$ & $29.8 \%$ & $41.1 \%$ & $25 \%$ & \\
\hline $32^{0 / 7}-36^{6 / 7}$ & $27.8 \%$ & $23.2 \%$ & $28.9 \%$ & \\
\hline $37^{0 / 7}-41^{6 / 7}$ & $35.5 \%$ & $23.2 \%$ & $41.7 \%$ & \\
\hline$>42$ weeks & $1.2 \%$ & $0 \%$ & $1.7 \%$ & \\
\hline Spontaneous preterm birth & $10.9 \%$ & $16.9 \%$ & $10.1 \%$ & 0.150 \\
\hline latrogenic preterm birth & $40.7 \%$ & $54.2 \%$ & $40.4 \%$ & 0.060 \\
\hline Birth weight, grams & $2215(1377-3128)$ & $1770(1045-2780)$ & $2510(1510-3230)$ & $<0.001$ \\
\hline Apgar at 5 min & $9(7-10)$ & $8(6-10)$ & $9(7-10)$ & 0.234 \\
\hline Umbilical pH & $7.26(7.20-7.31)$ & $7.26(7.21-7.31)$ & $7.26(7.19-7.31)$ & 0.729 \\
\hline Mortality & $17.2 \%$ & $43.1 \%$ & $9.7 \%$ & $<0.001$ \\
\hline Resuscitation & $5.1 \%$ & $11.1 \%$ & $3.4 \%$ & 0.057 \\
\hline Mechanical ventilation & $34.1 \%$ & $50 \%$ & $31.2 \%$ & 0.039 \\
\hline NICU admission & $45.1 \%$ & $50 \%$ & $45 \%$ & 0.600 \\
\hline Composite neonatal morbidity ${ }^{a}$ & $29.1 \%$ & $41.2 \%$ & $26.8 \%$ & 0.100 \\
\hline
\end{tabular}

Data in percentages and medians (Q1-Q3). $p$ values were calculated between antepartum and postpartum maternal ICU admission

NICUneonatal intensive care unit

${ }^{a}$ Composite neonatal morbidity was defined as the occurrence of respiratory distress syndrome, necrotizing enterocolitis, intraventricular hemorrhage, periventricular leukomalacia and/or sepsis 


\section{Discussion}

The mortality rate of young women after ICU admission in a tertiary care center varies strongly between different indications for admissions, of which cardiovascular disease is the most frequent. Women admitted during pregnancy or postpartum have a lower mortality rate as compared to non-obstetric patients in a similar age group ( $4.9 \%$ vs. $14 \%, p<0.001)$, despite higher APACHE II scores and a higher rate of mechanical ventilation.

The average Dutch ICU 1-year mortality rate is $22.6 \%$, but the average ICU population is predominantly male, $>60$ years old and has a median APACHE III score of 51 [10]. In our study young women have a better prognosis (mortality rate 13.3\%), although this seems to depend on admission diagnosis. Cardiovascular disease was the most prevalent diagnosis and was associated with a relatively low mortality of $6.2 \%$. The mortality rate in oncological patients was higher (23.9\%) as may be expected, but other subgroups also had markedly high case fatality rates (such as $19.6 \%$ for pulmonary disease). A possible explanation for the low mortality in cardiovascular disease might be the low-threshold use of intensive care facilities for hemodynamic monitoring of cardiac patients.

Women admitted to an ICU during pregnancy or postpartum have a lower mortality rate as compared to non-obstetric patients in a similar age group (4.9\% vs. $14 \%, p<0.001)$, despite higher APACHE II scores and a higher rate of mechanical ventilation. Relatively favorable outcome for obstetric admissions is in line with a single previous study on the subject (3.1\% vs. $19.6 \%$ ) [11]. The favorable outcome for the obstetric group may be partly explained by the direct obstetric causes and many pregnant women being previously physically healthy. Moreover, the physiological adaptations during pregnancy are evolutionary designed to limit blood loss and create additional reserves [12]. Instead of being a risk factor for adverse outcome, pregnancy might actually offer some physiological protection to the mother in critical illness. Additionally, there may be a high index of suspicion and carefulness in treating pregnant patients, which may create a low threshold for ICU admissions for less complex indications than in the non-obstetric group [7].

However, this is not evident from the APACHE II score. In contrast to mortality, the obstetric group has a higher APACHE II score than the non-obstetric group. The APACHE II score has previously been observed to overestimate mortality in obstetric populations, often explained by the altered physiological ranges in pregnancy $[11,13]$. However, the APACHE II normal ranges are often broad enough (heart rate 70-109; mean arterial pressure 70-109; respiratory rate 12-24) that even the physiologically altered values during pregnancy do not easily fall into the abnormal range [9, 14-16]. Other APACHE II parameters, such as the Glasgow Coma Score, are even more unlikely to be significantly influenced by pregnancy-induced changes. Recently, a pregnancy-specific risk model has been developed but this is not yet implemented in clinical care and cannot be used to compare obstetric and non-obstetric ICU admissions [6].

We found higher mechanical ventilation rates in the obstetric group as compared to the non-obstetric group. Particular care for respiratory complications should be taken in obstetric ICU care. The gravid uterus elevates the diaphragm, which combined with increased oxygen consumption and carbon dioxide production results in a decreased functional residual capacity [14].

The obstetric mortality rate $(4.9 \%)$ in this study is higher than reported by the two largest studies on obstetric ICU admissions (0.7-1.7\%), based on nationwide databases $[3,6]$. The difference in outcomes illustrates the higher complexity of the patient population in a tertiary referral center, as compared to a nationwide sample. Additionally, there was a high threshold for ICU admission in obstetric patients due to the presence of a dedicated level 2 Obstetric High Care unit. The latter accounted for an additional 2006 admissions during the study period, which suggests that dedicated obstetrical critical care units can substantially reduce ICU admissions, as part of these severely ill women might in another hospital have been admitted to the ICU [17].

The largest studies of obstetric ICU admissions do not describe maternal morbidity beyond ICU resource utilization $[3,6]$. We demonstrated that half of pregnant and postpartum patients required surgical or endovascular intervention, in addition to Caesarean section in $60.4 \%$. The most frequently performed interventions were uterine artery embolization and hysterectomy, which are commonly performed because of peripartum hemorrhage. Many of the procedures that were performed in this cohort (e.g. hysterectomy, colostomy, limb amputation) could be speculated to have significant impact on a woman's quality of life (QoL) [18]. In one study of QoL after obstetric ICU admission, 19\% of participants reported decreased QoL even after a median ICU LOS of $22 \mathrm{~h}$, which is considerably shorter than in our population [18]. The impact on QoL might be greater in a more severely ill population and warrants further research, which should include predictors of decreased QoL.

Perinatal death $(17.2 \%)$ is frequent, which is in line with earlier reports describing $13.6-34 \%$ perinatal mortality in critically ill mothers $[4,19,20]$. Perinatal mortality among women admitted antepartum was higher than in those admitted postpartum, which may be associated with the (often iatrogenic) higher rate of premature births [21]. 
Additionally, the direct obstetric complications (such as $\mathrm{PPH}$ ) seen predominantly in the postpartum admissions do not always affect the child. For antepartum admissions, extending the pregnancy duration may improve fetal outcome, but may also be detrimental to the mother and exposes the fetus to a potentially unfavorable environment created by maternal illness, such as acidosis or placental insufficiency [22]. The surviving infants had composite neonatal morbidity in $29.1 \%$ and NICU admission in $45.1 \%$, which exceeds the earlier described NICU admission rate of $11-36.7 \%$ among children born from critically ill mothers in smaller cohorts $[4,19]$.

Limitations of this study are its retrospective nature and possible heterogeneity because of advances in treatment during the 15-year timeframe. Our tertiary referral center is not representative for all ICU's, as we describe a high-risk population. However, this study has several strengths. We provide new insights into the causes of ICU admissions in young women, which are an often overlooked group. For obstetric admissions, we report relatively favorable mortality rates but high rates of interventions that may have lasting impact on quality of life, requiring further research. Our perinatal data may contribute to counseling on the expected fetal and neonatal complications after maternal ICU admission.

\section{Conclusions}

In this retrospective cohort study in a tertiary center, young women are admitted to the ICU most often for cardiovascular disease and their prognosis strongly depends on the primary indication for admission. Obstetric patients constitute a minor part of ICU admissions, and their mortality rate is lower compared to other young female ICU patients. This is despite higher need for invasive ventilation and higher APACHE II score.

\footnotetext{
Abbreviations

ICU: Intensive Care Unit; PPH: Postpartum hemorrhage; LOS: Length of stay; ECMO: Extracorporeal membrane oxygenation; NICU: Neonatal Intensive Care
} Unit; SD: Standard deviation.

\section{Acknowledgements}

None.

\section{Author contributions}

KPR, DG, CERMJ, KM, JJD, IKMR, AF, JWR-H and JMJC contributed to the conception and design of the study. Material preparation and data acquisition was performed by KPR, CERMJ and KM. Analysis and interpretation of the data was performed by KPR, KM and JMJC. The article was drafted by KPR and JMJC and all authors provided critical revision. All authors read and approved the final manuscript.

\section{Funding}

The authors received no financial support for the research, authorship and/or publication of this article.

\section{Availability of data and materials}

The raw data are available from the corresponding author KPR upon reasonable request. They are not publicly available due to them containing information that could compromise research participant privacy due to the nature of the study (rare and critical events in a single center during a defined time period). Individual consent to share individual data was not obtained according to national ethics guidelines for observational retrospective studies.

\section{Declarations}

\section{Ethics approval and consent to participate}

This study was performed in accordance with the ethical standards laid down in the 1964 Declaration of Helsinki and its later amendments. The Ethics Committee of the Erasmus University Medical Center has approved the research protocol and waived formal informed consent in view of the retrospective nature of the study, according to local national law and ethical guidelines (reference number MEC-2020-0056).

\section{Consent for publication}

Not applicable.

\section{Competing interests}

The authors declare that they have no competing interests.

\section{Author details}

'Department of Cardiology, Erasmus MC, University Medical Center Rotterdam, PO Box 2040, 3000 CA Rotterdam, The Netherlands. ${ }^{2}$ Department of Adult Intensive Care Medicine, Erasmus MC, University Medical Center Rotterdam, Rotterdam, The Netherlands. ${ }^{3}$ Department of Obstetrics and Gynecology, Erasmus MC - Sophia Children's Hospital, University Medical Center Rotterdam, Rotterdam, The Netherlands. ${ }^{4}$ Division of Neonatology, Department of Pediatrics, Erasmus MC - Sophia Children's Hospital, University Medical Center Rotterdam, Rotterdam, The Netherlands.

Received: 15 December 2020 Accepted: 31 May 2021

Published online: 19 June 2021

\section{References}

1. Wunsch H, Angus DC, Harrison DA, Linde-Zwirble WT, Rowan KM. Comparison of medical admissions to intensive care units in the United States and United Kingdom. Am J Respir Crit Care Med. 2011;183:1666-73.

2. Capuzzo M, Volta C, Tassinati T, Moreno R, Valentin A, Guidet B, et al. Hospital mortality of adults admitted to Intensive Care Units in hospitals with and without Intermediate Care Units: a multicentre European cohort study. Crit Care. 2014;18:551.

3. Maiden MJ, Finnis ME, Duke GJ, Huning E, Crozier T, Nguyen N, et al. Obstetric admissions to intensive care units in Australia and New Zealand: a registry-based cohort study. BJOG. 2020.

4. Cartin-Ceba R, Gajic O, lyer VN, Vlahakis NE. Fetal outcomes of critically ill pregnant women admitted to the intensive care unit for nonobstetric causes. Crit Care Med. 2008;36:2746-51.

5. Soubra SH, Guntupalli KK. Critical illness in pregnancy: an overview. Crit Care Med. 2005;33:S248-55.

6. Simpson NB, Shankar-Hari M, Rowan KM, Cecconi M, von Dadelszen P, Huning EY, et al. Maternal risk modeling in critical care-development of a multivariable risk prediction model for death and prolonged intensive care. Crit Care Med. 2020;48:663-72.

7. Sultan P, Arulkumaran N, Rhodes A. Provision of critical care services for the obstetric population. Best Pract Res Clin Obstet Gynaecol. 2013;27:803-9.

8. Edwards Z, Lucas DN, Gauntlett R. Is training in obstetric critical care adequate? An international comparison. Int J Obstet Anesth. 2019;37:96-105.

9. Knaus WA, Draper EA, Wagner DP, Zimmerman JE. APACHE II: a severity of disease classification system. Crit Care Med. 1985;13:818-29.

10. Stichting NICE. Dutch National Intensive Care Evaluation - Yearbook 2017. https://www.stichting-nice.nl/doc/jaarboek-2017-web.pdf (2018). Accessed 15 Feb 2020. 
11. Harrison DA, Penny JA, Yentis SM, Fayek S, Brady AR. Case mix, outcome and activity for obstetric admissions to adult, general critical care units: a secondary analysis of the ICNARC Case Mix Programme Database. Crit Care. 2005;9:\$25-37.

12. Mockridge AM. Physiology of pregnancy. Anaesth Intensive Care Med 2019;20:397-401.

13. Ryan HM, Sharma S, Magee LA, Ansermino JM, MacDonell K, Payne BA, et al. The usefulness of the APACHE II score in obstetric critical care: a structured review. J Obstet Gynaecol Can. 2016:38:909-18.

14. Rees GB, Broughton Pipkin F, Symonds EM, Patrick JM. A longitudinal study of respiratory changes in normal human pregnancy with crosssectional data on subjects with pregnancy-induced hypertension. Am J Obstet Gynecol. 1990;162:826-30.

15. Meah VL, Cockcroft JR, Backx K, Shave R, Stohr EJ. Cardiac output and related haemodynamics during pregnancy: a series of meta-analyses. Heart. 2016;102:518-26.

16. Melchiorre K, Sharma R, Thilaganathan B. Cardiac structure and function in normal pregnancy. Curr Opin Obstet Gynecol. 2012;24:413-21.

17. Zwart JJ, Dupuis JR, Richters A, Ory F, van Roosmalen J. Obstetric intensive care unit admission: a 2-year nationwide population-based cohort study. Intensive Care Med. 2010;36:256-63.
18. Seppanen P, Sund R, Ala-Kokko T, Roos M, Uotila J, Helminen M, et al. Obstetric patients' health-related quality of life before and after intensive care. Aust Crit Care. 2019;32:116-21.

19. De Greve M, Van Mieghem T, Van Den Berghe G, Hanssens M. Obstetric admissions to the intensive care unit in a Tertiary Hospital. Gynecol Obstet Invest. 2016;81:315-20.

20. Pollock W, Rose L, Dennis CL. Pregnant and postpartum admissions to the intensive care unit: a systematic review. Intensive Care Med. 2010;36:1465-74.

21. Vogel JP, Chawanpaiboon S, Moller AB, Watananirun K, Bonet M, Lumbiganon P. The global epidemiology of preterm birth. Best Pract Res Clin Obstet Gynaecol. 2018;52:3-12.

22. Aoyama K, Seaward PG, Lapinsky SE. Fetal outcome in the critically ill pregnant woman. Crit Care. 2014;18:307.

\section{Publisher's Note}

Springer Nature remains neutral with regard to jurisdictional claims in published maps and institutional affiliations.
Ready to submit your research? Choose BMC and benefit from:

- fast, convenient online submission

- thorough peer review by experienced researchers in your field

- rapid publication on acceptance

- support for research data, including large and complex data types

- gold Open Access which fosters wider collaboration and increased citations

- maximum visibility for your research: over $100 \mathrm{M}$ website views per year

At BMC, research is always in progress.

Learn more biomedcentral.com/submissions 